\title{
Nonhomogeneous initial and boundary
}

value problem for the Caputo-type fractional wave equation

\author{
Mehmet Ali Özarslan ${ }^{1 *}$ and Cemaliye Kürt ${ }^{1}$
}

\section{"Correspondence:}

mehmetali.ozarslan@emu.edu.tr

'Department of Mathematics,

Faculty of Arts and Sciences, Eastern

Mediterranean University,

Famagusta, North Cyprus, Turkey

\begin{abstract}
In this paper, we give an analytical solution of a fractional wave equation for a vibrating string with Caputo time fractional derivatives. We obtain the exact solution in terms of three parameter Mittag-Leffler function. Furthermore, some examples of the main result are exhibited.
\end{abstract}

MSC: 35L05; 26A33; 33E12; 44A10

Keywords: Fractional wave equation; Caputo time fractional derivative; Mittag-Leffler function; Laplace transform

\section{Introduction}

In recent years, fractional calculus has been one of the most popular topics in research [1-5]. There are many different definitions and representations of fractional integrals and derivatives in the literature, for instance, Riemann-Liouville integral, Riemann-Liouville derivatives, Caputo derivative, Hilfer derivative, and so on (see [6-16]).

The fractional calculus has been used effectively to solve different kinds of problems such as fractional relaxation and oscillation process, time fractional diffusive and wave processes [4, 17-21], generalized Langevin and fractional Fokker-Planck equations [2233]. Furthermore, many authors have obtained the solutions of time fractional diffusionwave equations in a bounded domain in terms of the Mittag-Leffler type functions (see $[22,31,34-44])$.

Here, we solve the following wave equation for a vibrating string:

$$
C_{*}^{\gamma} w(x, t)=\frac{\partial^{2} w(x, t)}{\partial x^{2}}-b C_{*}^{\alpha} w(x, t)+g(x, t)
$$

with Caputo time fractional derivatives $C_{*}^{\gamma}$ and $C_{*}^{\alpha}$ of orders $1<\gamma<2$ and $0<\alpha<1$, respectively, using the conditions

$$
\left.w(x, t)\right|_{x=0}=h_{1}(t),\left.\quad w(x, t)\right|_{x=l}=h_{2}(t)
$$

(c) The Author(s) 2019. This article is distributed under the terms of the Creative Commons Attribution 4.0 International License (http://creativecommons.org/licenses/by/4.0/), which permits unrestricted use, distribution, and reproduction in any medium, provided you give appropriate credit to the original author(s) and the source, provide a link to the Creative Commons license, and indicate if changes were made. 
and

$$
\left.w(x, t)\right|_{t=0_{+}}=\Theta(x),\left.\quad \frac{\partial w(x, t)}{\partial t}\right|_{t=0_{+}}=\Phi(x)
$$

where $t>0,0 \leq x \leq l, g(x, t), h_{1}(t), h_{2}(t), \Theta(x)$ and $\Phi(x)$ are sufficiently well-behaved functions, $b$ is a positive constant, $\tau$ is the memory time, and $g(x, t)$ is the external force.

This problem has the solutions for $x \in[0, l]$ and in $L(0, \infty)$ such that

$$
L(0, \infty)=\left\{f:\|f\|_{1}=\int_{0}^{\infty}|f(t)| d t<\infty\right\}
$$

where $L(0, \infty)$ the Lebesgue integrable function deals with $t$.

This paper is organized as follows. In Sect. 2, definitions and properties of Mittag-Leffler functions and fractional integrals and derivatives are presented. In Sect. 3, we consider the fractional wave equation (1) and solve this problem by using the separation of variables and Fourier expansion method. Also, some examples under the conditions are presented in Sect. 4. Finally, in Sect. 5, we give a concluding remark.

\section{Mathematical background}

\subsection{The Mittag-Leffler functions}

The Mittag-Leffler functions [45] were studied and introduced in the following series:

$$
\begin{aligned}
& E_{\alpha}(z)=\sum_{k=0}^{\infty} \frac{z^{k}}{\Gamma(\alpha k+1)}, \\
& (z \in \mathbb{C}, \operatorname{Re}(\alpha)>0) .
\end{aligned}
$$

A more general form of (4) was given by Wiman [46] in the form

$$
\begin{aligned}
& E_{\alpha, \beta}(z)=\sum_{k=0}^{\infty} \frac{z^{k}}{\Gamma(\alpha k+\beta)}, \\
& (z, \beta \in \mathbb{C}, \operatorname{Re}(\alpha)>0) .
\end{aligned}
$$

It is obvious that, by using (4) and (5), we have $E_{\alpha, 1}(z)=E_{\alpha}(z)$. The Mittag-Leffler functions are a generalization of the exponential, hyperbolic, and trigonometric functions since $E_{1,1}(z)=e^{z}, E_{2,1}\left(z^{2}\right)=\cosh (z), E_{2,1}\left(-z^{2}\right)=\cos (z)$, and $E_{2,2}\left(-z^{2}\right)=\sin (z) / z$.

The generalized Mittag-Leffler functions were defined by Praphakar [47], that is,

$$
\begin{aligned}
& E_{\alpha, \beta}^{\gamma}(z)=\sum_{k=0}^{\infty} \frac{(\gamma)_{k}}{\Gamma(\alpha k+\beta)} \frac{z^{k}}{k !}, \\
& (\alpha, \beta, \gamma \in \mathbb{C}, \operatorname{Re}(\alpha)>0, \operatorname{Re}(\beta)>0 ; z \in \mathbb{C}),
\end{aligned}
$$

where $(\gamma)_{k}$ is the Pochhammer symbol [48] defined by

$$
(\gamma)_{k}=\frac{\Gamma(\gamma+k)}{\Gamma(\gamma)}= \begin{cases}1 ; & k=0, \gamma \neq 0, \\ \gamma(\gamma+1) \cdots(\gamma+k-1) ; & k=1,2, \ldots\end{cases}
$$


Note that $E_{\alpha, \beta}^{1}(x)=E_{\alpha, \beta}(x)$. The four parameter Mittag-Leffler function [49] was defined by

$$
\begin{aligned}
& E_{\alpha, \beta}^{\gamma, \kappa}(z)=\sum_{n=0}^{\infty} \frac{(\gamma)_{\kappa n}}{\Gamma(\alpha n+\beta)} \frac{z^{n}}{n !}, \\
& (\alpha, \beta, \gamma \in \mathbb{C}, \operatorname{Re}(\alpha)>\max \{0, \operatorname{Re}(\kappa)-1\}, \operatorname{Re}(\kappa)>0) .
\end{aligned}
$$

From (6) and (7), we see that $E_{\alpha, \beta}^{\gamma, 1}(z)=E_{\alpha, \beta}^{\gamma}(z)$.

The Laplace transform of the Mittag-Leffler functions (6) is represented by (see $[47,50]$ )

$$
\mathbb{L}\left[t^{\beta-1} E_{\alpha, \beta}^{\gamma}\left(u t^{\alpha}\right)\right](s)=\frac{s^{\alpha \gamma-\beta}}{\left(s^{\alpha}-u\right)^{\gamma}},
$$

where $\left|\frac{u}{s^{\alpha}}\right|<1$.

Now, we give basic definitions and properties that will be used throughout the paper.

Definition 2.1 (Riemann-Liouville integral (see [5])) Let $\Omega=[a, b]$ be a finite interval of the real axis. The Riemann-Liouville fractional integral of order $\mu \in \mathbb{C}(\operatorname{Re}(\mu)>0)$ is defined by

$$
{ }_{x} I_{a^{+}}^{\gamma}[g]=\frac{1}{\Gamma(\gamma)} \int_{a}^{x} \frac{g(t) d t}{(x-t)^{1-\gamma}} \quad(x>a, \operatorname{Re}(\mu)>0) .
$$

Definition 2.2 (Caputo derivative (see [4] and [51])) Let $\gamma>0, n=\lceil\gamma\rceil$, and $g \in A C^{n}[a, b]$. The Caputo derivative of $\gamma>0$ is defined as

$$
C_{*}^{\gamma} g(t)= \begin{cases}\frac{1}{\Gamma(n-\gamma)} \int_{0}^{t} \frac{g^{(n)}(s)}{(t-s)^{\gamma+1-n}} d s ; & n-1<\gamma<n, \\ \frac{d^{n} g(t)}{d t^{n}} ; & \gamma=n .\end{cases}
$$

Note that the following expression gives us the relationship between the Caputo fractional derivative (10) and the Riemann-Liouville fractional integral operator (9) (see [4])

$$
C_{*}^{\gamma} g(t)=I_{0^{+}}^{n-\gamma} g^{(n)}(t)
$$

where $g^{(n)}$ is denoted by $n$-order derivative.

We give the Laplace transform for the Caputo fractional derivative in the following formula (see $[5,52])$ :

$$
\mathbb{L}\left[C_{*}^{\gamma} g(t)\right](s)=\int_{0}^{\infty} e^{-s t} C_{*}^{\gamma} g(t) d t=s^{\gamma} G(s)-\sum_{k=0}^{n-1} g^{(k)}\left(0_{+}\right) s^{\gamma-1-k},
$$

where $\gamma \in(n-1, n)$ and $G(s)$ is the representation of Laplace transform for the function $g(t)$. Clearly, $C_{*}^{\gamma} 1 \equiv 0$ for $\gamma>0$. 
Definition 2.3 The integral operator $\mathcal{E}_{a^{+} ; \alpha, \beta}^{u ; \gamma} \varphi$ (see [49]) was introduced by Srivastava and Tomovski in the following form:

$$
\begin{aligned}
& \left(\mathcal{E}_{a^{+} ; \alpha, \beta}^{u ; \gamma, \kappa} \varphi\right)(x)=\int_{a}^{x}(x-t)^{\beta-1} E_{\alpha, \beta}^{\gamma, \kappa}\left[u(x-t)^{\alpha}\right] \varphi(t) d t, \\
& (\rho, \mu, u, \gamma \in \mathbb{C}, \operatorname{Re}(\rho), \operatorname{Re}(\mu)>0),
\end{aligned}
$$

where $E_{\alpha, \beta}^{\gamma, \kappa}(z)$ is the four parameter Mittag-Leffler function given in (7).

When $u=0$ and $a=0$, the integral operator (12) coincides with the Riemann-Liouville integral operator (9) such that

$$
\left(\mathcal{E}_{0^{+} ; \alpha, \beta}^{0 ; \gamma} \varphi\right)(x)=\left(I_{0^{+}}^{\beta} \varphi\right)(x)
$$

\section{Analytical results for the problem}

In this section, we investigate the analytical solution of the proposed problem (1)-(3). In order to obtain the solution, we need the following lemmas.

Lemma 3.1 Let $s, b, \alpha, \lambda_{n} \in \mathbb{R}^{+}$and $u \in \mathbb{R}$. Then the inverse Laplace transform of the function

$$
f(s)=\frac{s^{\gamma-1}+b s^{\alpha-1}+u s^{\gamma-2}}{s^{\gamma}+b s^{\alpha}+\lambda_{n}}
$$

deals with

$$
\begin{aligned}
\left(\mathbb{L}^{-1} f\right)(t)= & \mathbb{L}^{-1}[f(s)](t) \\
= & \sum_{p=0}^{\infty}(-b)^{p} t^{(\gamma-\alpha) p} E_{\gamma,(\gamma-\alpha) p+1}^{(p+1)}\left(-\lambda_{n} t^{\gamma}\right) \\
& +b \sum_{p=0}^{\infty}(-b)^{p} t^{(\gamma-\alpha)(p+1)} E_{\gamma,(\gamma-\alpha)(p+1)+1}^{(p+1)}\left(-\lambda_{n} t^{\gamma}\right) \\
& +u \sum_{p=0}^{\infty}(-b)^{p} t^{(\gamma-\alpha) p+1} E_{\gamma,(\gamma-\alpha) p+2}^{(p+1)}\left(-\lambda_{n} t^{\gamma}\right),
\end{aligned}
$$

where $0<\frac{\lambda_{n}}{s^{\gamma}+b s^{\alpha}}<1$ and $0<\frac{b}{s^{\gamma-\alpha}}<1$,

Proof Since $0<\frac{\lambda_{n}}{s^{\gamma}+b s^{\alpha}}<1$ and $0<\frac{b}{s^{\gamma-\alpha}}<1$, we rewrite relation (13) in the following way:

$$
\begin{aligned}
f(s) & =\left(s^{\gamma-1}+b s^{\alpha-1}+u s^{\gamma-2}\right) \cdot \frac{s^{-\alpha}}{s^{\gamma-\alpha}+b} \cdot \frac{1}{1+\frac{\lambda_{n} s^{-\alpha}}{s^{\gamma-\alpha}+b}} \\
& =\sum_{j=0}^{\infty}\left(-\lambda_{n}\right)^{j}\left\{\frac{s^{-\alpha(j+1)+\gamma-1}}{\left(s^{\gamma-\alpha}+b\right)^{j+1}}+b \frac{s^{-\alpha j-1}}{\left(s^{\gamma-\alpha}+b\right)^{j+1}}+u \frac{s^{-\alpha(j+1)+\gamma-2}}{\left(s^{\gamma-\alpha}+b\right)^{j+1}}\right\} .
\end{aligned}
$$


By using relation (8), we get

$$
\begin{aligned}
& \mathbb{L}^{-1}[f(s)](t) \\
& =\sum_{j=0}^{\infty}\left(-\lambda_{n}\right)^{j} t^{\gamma j} E_{\gamma-\alpha, \gamma j+1}^{(j+1)}\left(-b t^{\gamma-\alpha}\right) \\
& +b \sum_{j=0}^{\infty}\left(-\lambda_{n}\right)^{j} t^{\gamma(j+1)-\alpha} E_{\gamma-\alpha, \gamma(j+1)-\alpha+1}^{(j+1)}\left(-b t^{\gamma-\alpha}\right) \\
& +u \sum_{j=0}^{\infty}\left(-\lambda_{n}\right)^{j} t^{\gamma j+1} E_{\gamma-\alpha, \gamma j+2}^{j+1}\left(-b t^{\gamma-\alpha}\right) \\
& =\sum_{j=0}^{\infty} \sum_{p=0}^{\infty}\left(-\lambda_{n}\right)^{j} t^{\gamma j} \frac{(j+1)_{p}}{\Gamma((\gamma-\alpha) p+\gamma j+1)} \frac{\left(-b t^{\gamma-\alpha}\right)^{p}}{p !} \\
& +b \sum_{j=0}^{\infty} \sum_{p=0}^{\infty}\left(-\lambda_{n}\right)^{j} t^{\gamma j+1-\alpha} \frac{(j+1)_{p}}{\Gamma((\gamma-\alpha) p+\gamma(j+1)-\alpha+1)} \frac{\left(-b t^{\gamma-\alpha}\right)^{p}}{p !} \\
& +u \sum_{j=0}^{\infty} \sum_{p=0}^{\infty}\left(-\lambda_{n}\right)^{j} t^{\gamma j+1} \frac{(j+1)_{p}}{\Gamma((\gamma-\alpha) p+\gamma j+2)} \frac{\left(-b t^{\gamma-\alpha}\right)^{p}}{p !} \\
& =\sum_{j=0}^{\infty} \sum_{p=0}^{\infty}(-b)^{p} t^{(\gamma-\alpha) p} \frac{(p+1)_{j}}{\Gamma((\gamma-\alpha) p+\gamma j+1)} \frac{\left(-\lambda_{n} t^{\gamma}\right)^{j}}{j !} \\
& +b \sum_{j=0}^{\infty} \sum_{p=0}^{\infty}(-b)^{p} t^{(\gamma-\alpha)(p+1)} \frac{(p+1)_{j}}{\Gamma(\gamma j+(\gamma-\alpha)(p+1)+1)} \frac{\left(-\lambda_{n} t^{\gamma}\right)^{j}}{j !} \\
& +u \sum_{j=0}^{\infty} \sum_{p=0}^{\infty}(-b)^{p} t^{(\gamma-\alpha) p+1} \frac{(p+1)_{j}}{\Gamma((\gamma-\alpha) p+\gamma j+2)} \frac{\left(-\lambda_{n} t^{\gamma}\right)^{j}}{j !} \\
& =\sum_{p=0}^{\infty}(-b)^{p} t^{(\gamma-\alpha) p} E_{\gamma,(\gamma-\alpha) p+1}^{(p+1)}\left(-\lambda_{n} t^{\gamma}\right) \\
& +b \sum_{p=0}^{\infty}(-b)^{p} t^{(\gamma-\alpha)(p+1)} E_{\gamma,(\gamma-\alpha)(p+1)+1}^{(p+1)}\left(-\lambda_{n} t^{\gamma}\right) \\
& +u \sum_{p=0}^{\infty}(-b)^{p} t^{(\gamma-\alpha) p+1} E_{\gamma,(\gamma-\alpha) p+2}^{(p+1)}\left(-\lambda_{n} t^{\gamma}\right)
\end{aligned}
$$

Thus, we get the desired result.

Lemma 3.2 Let $s, b, \alpha, \lambda_{n} \in \mathbb{R}^{+}$. We have

$$
\begin{aligned}
& \mathbb{L}^{-1}\left[\frac{1}{s^{\gamma}+b s^{\alpha}+\lambda_{n}} \mathbb{L}\left[\widetilde{g}_{n}(t)\right](s)\right](t)=\sum_{p=0}^{\infty}(-b)^{p}\left(\mathcal{E}_{0^{+} ; \gamma,(\gamma-\alpha) p+\gamma}^{-\lambda_{n} ; p+1,1} \widetilde{g}_{n}\right)(t), \\
& \left(0<\frac{\lambda_{n}}{s^{\gamma}+b s^{\alpha}}<1,0<\frac{b}{s^{\gamma-\alpha}}<1\right),
\end{aligned}
$$

where $\mathcal{E}_{0^{+} ; \gamma,(\gamma-\alpha) p+\gamma}^{-\lambda_{n} ; p+1,1}$ is given in (12) and $\widetilde{g}_{n}(t)$ is a given function. 
Proof Let

$$
h(s)=\frac{1}{s^{\gamma}+b s^{\alpha}+\lambda_{n}} \mathbb{L}\left[\widetilde{g}_{n}(t)\right](s)
$$

We rewrite relation (15) in the following form:

$$
h(s)=\frac{s^{-\alpha}}{s^{\gamma-\alpha}+b} \cdot \frac{1}{1+\frac{\lambda_{n} s^{-\alpha}}{s^{\gamma-\alpha}+b}} \mathbb{L}\left[\widetilde{g}_{n}(t)\right](s) .
$$

Since $0<\frac{\lambda_{n}}{s^{\gamma}+b s^{\alpha}}<1$, we have

$$
h(s)=\sum_{j=0}^{\infty}\left(-\lambda_{n}\right)^{j} \frac{s^{-\alpha(j+1)}}{\left(s^{\gamma-\alpha}+b\right)^{j+1}} \mathbb{L}\left[\widetilde{g}_{n}(t)\right](s) .
$$

With the help of relation (8), we obtain

$$
\begin{aligned}
h(s) & =\mathbb{L}\left[\sum_{j=0}^{\infty}\left(-\lambda_{n}\right)^{j} t^{\gamma(j+1)-1} E_{\gamma-\alpha, \gamma(j+1)}^{j+1}\left(-b t^{\gamma-\alpha}\right)\right](s) \mathbb{L}\left[\widetilde{g}_{n}(t)\right](s) \\
& =\mathbb{L}\left[\sum_{p=0}^{\infty}(-b)^{p} t^{(\gamma-\alpha) p+\gamma-1} E_{\gamma,(\gamma-\alpha) p+\gamma}^{p+1}\left(-\lambda_{n} t^{\gamma}\right)\right](s) \mathbb{L}\left[\widetilde{g}_{n}(t)\right](s) .
\end{aligned}
$$

Applying the Parseval theorem for the Laplace transform (see [53])

$$
\mathbb{L}\left[\int_{0}^{x} k(x-t) \varphi(t) d t\right](s)=\mathbb{L}[k(x)](s) \mathbb{L}[\varphi(x)](s),
$$

we have

$$
h(s)=\sum_{p=0}^{\infty}(-b)^{p} \mathbb{L}\left[\int_{0}^{t}(t-\tau)^{(\gamma-\alpha) p+\gamma-1} E_{\gamma,(\gamma-\alpha) p+\gamma}^{p+1}\left(-\lambda_{n}[t-\tau]^{\gamma}\right) \widetilde{g}_{n}(\tau)\right](s) .
$$

Taking the inverse Laplace transform on both sides of (16), we get

$$
\begin{aligned}
\mathbb{L}^{-1} & {[g(s)](t) } \\
\quad= & \mathbb{L}^{-1}\left[\frac{1}{s^{\gamma}+b s^{\alpha}+\lambda_{n}} \mathbb{L}\left[\widetilde{g}_{n}(t)\right](s)\right](t)=\sum_{p=0}^{\infty}(-b)^{p}\left(\mathcal{E}_{0^{+} ; \gamma,(\gamma-\alpha) p+\gamma}^{-\lambda_{n} ; p+1,1} \widetilde{g}_{n}\right)(t),
\end{aligned}
$$

which is the desired result.

The solution of the problem given by (1)-(3) is given in the following theorem. 
Theorem 3.3 The problem given in (1), (2), and (3) has a summable solution in $L(0, \infty)$ with respect to $t$ as follows:

$$
\begin{aligned}
w(x, t)= & \sum_{n=1}^{\infty} T_{n}(t) \sin \left(\frac{n \pi x}{l}\right) \\
& +\sum_{n=1}^{\infty} \sum_{p=0}^{\infty}(-b)^{p}\left(\mathcal{E}_{0^{+} ; \gamma, \gamma(\gamma-\alpha) p+\gamma}^{-\lambda_{n} ; p+1,1} \widetilde{g}_{n}\right)(t) \sin \left(\frac{n \pi x}{l}\right)+h_{1}(t)+\frac{x}{l}\left[h_{2}(t)-h_{1}(t)\right]
\end{aligned}
$$

for $x \in[0, l]$, where

$$
\begin{aligned}
T_{n}(t)= & T_{n}^{(0)}\left(0_{+}\right)\left[\sum_{p=0}^{\infty}(-b)^{p} t^{(\gamma-\alpha) p} E_{\gamma,(\gamma-\alpha) p+1}^{(p+1)}\left(-\frac{n^{2} \pi^{2}}{l^{2}} t^{\gamma}\right)\right. \\
& +b \sum_{p=0}^{\infty}(-b)^{p} t^{(\gamma-\alpha)(p+1)} E_{\gamma,(\gamma-\alpha)(p+1)+1}^{(p+1)}\left(-\frac{n^{2} \pi^{2}}{l^{2}} t^{\gamma}\right) \\
& \left.+\frac{T_{n}^{(1)}\left(0_{+}\right)}{T_{n}^{(0)}\left(0_{+}\right)} \sum_{p=0}^{\infty}(-b)^{p} t^{(\gamma-\alpha) p+1} E_{\gamma,(\gamma-\alpha)+2}^{(p+1)}\left(-\frac{n^{2} \pi^{2}}{l^{2}} t^{\gamma}\right)\right], \\
\widetilde{g}_{n}(t)= & \frac{2}{l} \int_{0}^{l}\left[g(x, t)+\frac{\partial^{2}\left(h_{1}(t)+\frac{x}{l}\left[h_{2}(t)-h_{1}(t)\right]\right)}{\partial x^{2}}\right. \\
& \left.-C_{*}^{\gamma}\left(h_{1}(t)+\frac{x}{l}\left[h_{2}(t)-h_{1}(t)\right]\right)-b C_{*}^{\alpha} w(x, t)\right] \sin \left(\frac{n \pi x}{l}\right) d x, \\
T_{n}^{(0)}\left(0_{+}\right) & =\frac{2}{l} \int_{0}^{l}\left(\widetilde{\Theta}(x)-\left.\left(h_{1}(t)+\frac{x}{l}\left[h_{2}(t)-h_{1}(t)\right]\right)\right|_{t=0_{+}}\right) \sin \left(\frac{n \pi x}{l}\right) d x,
\end{aligned}
$$

and

$$
T_{n}^{(1)}\left(0_{+}\right)=\frac{2}{l} \int_{0}^{l}\left(\widetilde{\Phi}(x)-\left.\frac{\partial\left(h_{1}(t)+\frac{x}{l}\left[h_{2}(t)-h_{1}(t)\right]\right)}{\partial t}\right|_{t=0_{+}}\right) \sin \left(\frac{n \pi x}{l}\right) d x .
$$

Proof Suppose $w(x, t)$ is given as

$$
w(x, t)=W(x, t)+v(x, t) .
$$

Clearly, conditions (2) satisfy $v(x, t)$ where

$$
v(x, t)=h_{1}(t)+\frac{x}{l}\left[h_{2}(t)-h_{1}(t)\right] .
$$

From relations (18) and (19), we get

$$
\left.W(x, t)\right|_{x=0}=0,\left.\quad W(x, t)\right|_{x=l}=0 .
$$

By (3), we get

$$
\left.W(x, t)\right|_{t=0_{+}}=\Theta(x)-\left.v(x, t)\right|_{t=0_{+}}=\widetilde{\Theta}(x)
$$




$$
\left.\frac{\partial W(x, t)}{\partial t}\right|_{t=0_{+}}=\Phi(x)-\left.\frac{\partial v(x, t)}{\partial x}\right|_{t=0_{+}}=\widetilde{\Phi}(x) .
$$

By representing

$$
W(x, t)=W_{1}(x, t)+W_{2}(x, t)
$$

and by using (1) and (18), we get

$$
C_{*}^{\gamma}\left[W_{1}(x, t)+W_{2}(x, t)\right]=\frac{\partial^{2}}{\partial x^{2}}\left[W_{1}(x, t)+W_{2}(x, t)\right]-b C_{*}^{\alpha}\left[W_{1}(x, t)+W_{2}(x, t)\right]+\widetilde{g}(x, t),
$$

where

$$
\widetilde{g}(x, t)=g(x, t)+\frac{\partial^{2} v(x, t)}{\partial x^{2}}-C_{*}^{\gamma} v(x, t)-b C_{*}^{\alpha} w(x, t) .
$$

The problem now can be reduced as follows:

$$
\begin{aligned}
& C_{*}^{\gamma} W_{1}(x, t)=\frac{\partial^{2} W_{1}(x, t)}{\partial x^{2}}-b W_{1}(x, t), \\
& \left.W_{1}(x, t)\right|_{x=0}=0,\left.\quad W_{1}(x, t)\right|_{x=l}=0, \\
& \left.W_{1}(x, t)\right|_{t=0_{+}}=\widetilde{\Theta}(x),\left.\quad \frac{\partial W_{1}(x, t)}{\partial t}\right|_{t=0_{+}}=\widetilde{\Phi}(x),
\end{aligned}
$$

and

$$
\begin{aligned}
& C_{*}^{\gamma} W_{2}(x, t)=\frac{\partial^{2} W_{2}(x, t)}{\partial x^{2}}-b C_{*}^{\alpha} W_{2}(x, t)+\tilde{g}(x, t), \\
& \left.W_{2}(x, t)\right|_{x=0}=0,\left.\quad W_{2}(x, t)\right|_{x=l}=0, \\
& \left.W_{2}(x, t)\right|_{t=0_{+}}=0,\left.\quad \frac{\partial W_{2}(x, t)}{\partial t}\right|_{t=0_{+}}=0 .
\end{aligned}
$$

Letting $W_{1}(x, t)=X(x) T(t)$, the differential equations take the following forms:

$$
\begin{aligned}
& C_{*}^{\gamma} T(t)+b C_{*}^{\alpha} T(t)+\lambda T(t)=0, \\
& \frac{d^{2} X(x)}{d x^{2}}+\lambda X(x)=0,
\end{aligned}
$$

where $\lambda$ is called a separation constant. So, the solution of the Sturm-Liouville problem (25) deals with the function $X(x)$ with boundary conditions:

$$
\left.X(x)\right|_{x=0}=0,\left.\quad X(x)\right|_{x=l}=0 .
$$

The eigenfunctions of the problem are given in the form $X_{n}(x)=\sin \left(\sqrt{\lambda_{n}} x\right)$ where $\lambda_{n}=$ $\frac{n^{2} \pi^{2}}{l^{2}},\left(0<\lambda_{1}<\lambda_{2}<\cdots<\lambda_{n} \cdots\right)$. The relation for the eigenfunctions is satisfied by

$$
\int_{0}^{l} X_{n}^{2}(x) d x=\left\|X_{n}\right\|^{2} \delta_{n m}
$$

where $\left\|X_{n}\right\|^{2}=\frac{1}{2}$ is the norm of the eigenfunctions and $\delta_{n m}$ is the Kronecker delta. 
By using the Laplace transform, (24) is solved in the space $L(0, \infty)$. So, we get

$$
\begin{aligned}
s^{\gamma} & \mathbb{L}\left[T_{n}(t)\right](s)-s^{\gamma-1} T_{n}^{(0)}\left(0_{+}\right)-s^{\gamma-2} T_{n}^{(1)}\left(0_{+}\right) \\
& +b\left\{s^{\alpha} \mathbb{L}\left[T_{n}(t)\right](s)-s^{\alpha-1} T_{n}^{(0)}\left(0_{+}\right)\right\} \\
& +\lambda_{n} \mathbb{L}\left[T_{n}(t)\right](s)=0 .
\end{aligned}
$$

From (27), we get

$$
\mathbb{L}\left[T_{n}(t)\right](s)=T_{n}^{(0)}\left(0_{+}\right)\left[\frac{s^{\gamma-1}+b s^{\alpha-1}+\frac{T_{n}^{(1)}\left(0_{+}\right)}{T_{n}^{(0)}\left(0_{+}\right)} s^{\gamma-2}}{s^{\gamma}+b s^{\alpha}+\lambda_{n}}\right] .
$$

By using (14) and Lemma 3.1, the inverse Laplace transform of relation (28) yields

$$
\begin{aligned}
& T_{n}(t) \\
& =T_{n}^{(0)}\left(0_{+}\right)\left[\sum_{p=0}^{\infty}(-b)^{p} t^{(\gamma-\alpha) p} E_{\gamma,(\gamma-\alpha) p+1}^{(p+1)}\left(-\lambda_{n} t^{\gamma}\right)\right. \\
& \quad+b \sum_{p=0}^{\infty}(-b)^{p} t^{(\gamma-\alpha)(p+1)} E_{\gamma,(\gamma-\alpha)(p+1)+1}^{(p+1)}\left(-\lambda_{n} t^{\gamma}\right) \\
& \left.\quad+\frac{T_{n}^{(1)}\left(0_{+}\right)}{T_{n}^{(0)}\left(0_{+}\right)} \sum_{p=0}^{\infty}(-b)^{p} t^{(\gamma-\alpha) p+1} E_{\gamma,(\gamma-\alpha) p+2}^{(p+1)}\left(-\lambda_{n} t^{\gamma}\right)\right],
\end{aligned}
$$

so we obtain the solution of $W_{1}(x, t)$ such that

$$
W_{1}(x, t)=\sum_{n=1}^{\infty} T_{n}(t) \sin \left(\frac{n \pi x}{l}\right) .
$$

By using the Fourier expansions, we find the solution of (21):

$$
\begin{aligned}
& W_{2}(x, t)=\sum_{n=1}^{\infty} w_{n}(t) \sin \left(\frac{n \pi x}{l}\right), \\
& \widetilde{g}(x, t)=\sum_{n=1}^{\infty} \widetilde{g}_{n}(t) \sin \left(\frac{n \pi x}{l}\right),
\end{aligned}
$$

where $\widetilde{g}_{n}(t)$ is represented in (17). From (30), (31), and (21), we get

$$
\sum_{n=1}^{\infty}\left[C_{*}^{\gamma} w_{n}(t)+b C_{*}^{\alpha} w_{n}(t)+\lambda_{n} w_{n}(t)-\widetilde{g}_{n}(t)\right] \sin \left(\frac{n \pi x}{l}\right)=0
$$

if

$$
C_{*}^{\gamma} w_{n}(t)+b C_{*}^{\alpha} w_{n}(t)+\lambda_{n} w_{n}(t)-\widetilde{g}_{n}(t)=0,
$$

where $n \in \mathbb{N}$. 
Using the Laplace transform method (11) to (32), we get

$$
\begin{aligned}
& \left.s^{\gamma} \mathbb{L}\left[w_{n}(t)\right](s)-s^{\gamma-1} w_{n}^{(0)}\left(0_{+}\right)-s^{\alpha-2} w_{n}^{(1)}\left(0_{+}\right)\right] \\
& +b\left\{s^{\alpha} \mathbb{L}\left[w_{n}(t)\right](s)-s^{\alpha-1} w_{n}\left(0_{+}\right)\right\} \\
& \quad+\lambda_{n} \mathbb{L}\left[w_{n}(t)\right](s)-\mathbb{L}\left[\widetilde{g}_{n}(t)\right](s)=0 .
\end{aligned}
$$

From conditions (23), it follows that $\left.\frac{\partial^{p} w_{n}(x, t)}{\partial t^{p}}\right|_{t=0^{+}}=0$ for $p=0$, 1. From (33), we get

$$
\mathbb{L}\left[w_{n}(t)\right](s)=\frac{1}{s^{\gamma}+b s^{\alpha}+\lambda_{n}} \mathbb{L}\left[\widetilde{g}_{n}(t)\right](s) .
$$

Finally, we get the inverse Laplace transform of (34) and use Lemma 3.2 to obtain the following result:

$$
w_{n}(t)=\sum_{p=0}^{\infty}(-b)^{p}\left(\mathcal{E}_{0^{+} ; \gamma,(\gamma-\alpha) p+\gamma}^{-\lambda_{n} ; p+1,1} \widetilde{g}_{n}\right)(t) .
$$

Thus, the proof is completed.

\section{Some applications of the main problem}

In this section, we give some applications for time fractional wave equation (1)-(3) by considering special cases of the external force, conditions given in (2) and (3).

Example 4.1 Let $g(x, t)=0, \Theta(x)=x(1-x), \Phi(x)=0, h_{1}(t)=h_{2}(t)=0, x \in[0,1]$ in the above theorem. The time fractional wave equation takes the form as follows:

$$
C_{*}^{\gamma} w(x, t)=\frac{\partial^{2} w(x, t)}{\partial x^{2}}-b C_{*}^{\alpha} w(x, t)
$$

where $1<\gamma<2$ and $0<\alpha<1$, with the conditions

$$
\left.w(x, t)\right|_{x=0}=0,\left.\quad w(x, t)\right|_{x=1}=0
$$

and

$$
\left.w(x, t)\right|_{t=0_{+}}=0,\left.\quad \frac{\partial w(x, t)}{\partial t}\right|_{t=0_{+}}=0
$$

has the following solution

$$
w(x, t)=\sum_{n=1}^{\infty} T_{n}(t) \sin (n \pi x),
$$


where

$$
\begin{aligned}
T_{n}(t)= & 4 \frac{1-(-1)^{n}}{n^{3} \pi^{3}}\left[\sum_{p=0}^{\infty}(-b)^{p} t^{(\gamma-\alpha) p} E_{\gamma,(\gamma-\alpha) p+1}^{(p+1)}\left(-n^{2} \pi^{2} t^{\gamma}\right)\right. \\
& +b \sum_{p=0}^{\infty}(-b)^{p} t^{(\gamma-\alpha)(p+1)} E_{\gamma,(\gamma-\alpha)(p+1)+1}^{(p+1)}\left(-n^{2} \pi^{2} t^{\gamma}\right) \\
& \left.+u \sum_{p=0}^{\infty}(-b)^{p} t^{(\gamma-\alpha) p+1} E_{\gamma,(\gamma-\alpha) p+2}^{(p+1)}\left(-n^{2} \pi^{2} t^{\gamma}\right)\right] .
\end{aligned}
$$

When $n=2 r(r=1,2, \ldots)$, we have $T_{2 r}(t)=0$. Therefore, we have just the odd terms $T_{2 r-1}(t)$. Hence, the solution of (36) is $w(x, t)=\sum_{r=1}^{\infty} T_{2 r-1}(t) \sin [(2 r-1) \pi x]$.

Example 4.2 Let $g(x, t)=c t^{\kappa-1} E_{\alpha, \kappa}^{\zeta}\left(-t^{\alpha}\right), b=1, \tau=1, \Theta(x)=x(1-x), \Phi(x)=0, h_{1}(t)=$ $h_{2}(t)=0, x \in[0,1]$ in the above theorem. The time fractional wave equation takes the following form:

$$
C_{*}^{\gamma} w(x, t)=\frac{\partial^{2} w(x, t)}{\partial x^{2}}-b C_{*}^{\alpha} w(x, t)+c t^{\kappa-1} E_{\alpha, \kappa}^{\zeta}\left(-t^{\alpha}\right),
$$

where $1<\gamma<2,1<\alpha<2, c$ is any constant, with the conditions

$$
\left.w(x, t)\right|_{x=0}=0,\left.\quad w(x, t)\right|_{x=1}=0
$$

and

$$
\left.w(x, t)\right|_{t=0_{+}}=0,\left.\quad \frac{\partial w(x, t)}{\partial t}\right|_{t=0_{+}}=0
$$

has the following solution

$$
w(x, t)=\sum_{n=1}^{\infty} T_{n}(t) \sin (n \pi x)+\sum_{n=1}^{\infty} w_{n}(t) \sin (n \pi x)
$$

where $T_{n}(t)$ is given by (37), and

$$
w_{n}(t)=2 c \frac{\left\{1-(-1)^{n}\right\}}{n \pi} \sum_{p=0}^{\infty}(-b)^{p} t^{\mu+\kappa-1} E_{\rho, \mu+\kappa}^{\gamma+\zeta}\left(-t^{\rho}\right) .
$$

Note that only odd terms $T_{2 r-1}(t)$ and $U_{2 r-1}(t)$ are not equal to zero for $r=1,2, \ldots$

\section{Concluding remark}

For $\gamma \rightarrow 2$, (1) becomes

$$
\frac{\partial^{2} w(x, t)}{\partial t^{2}}=\frac{\partial^{2} w(x, t)}{\partial x^{2}}-b C_{*}^{\alpha} w(x, t)+g(x, t),
$$

with conditions

$$
\left.w(x, t)\right|_{x=0}=h_{1}(t),\left.\quad w(x, t)\right|_{x=l}=h_{2}(t),
$$


and

$$
\left.w(x, t)\right|_{t=0_{+}}=\Theta(x),\left.\quad \frac{\partial w(x, t)}{\partial t}\right|_{t=0_{+}}=\Phi(x)
$$

which is considered in [43].

The above problem has the solution (see p. 1558, [43], (27)-(29)) $w(x, t)=W_{1}(x, t)+$ $W_{2}(x, t)+v(x, t)$, with

$$
\begin{aligned}
& W_{1}(x, t)=\sum_{n=1}^{\infty}\left\{\sum_{p=0}^{\infty}(-b)^{p} t^{(2-\alpha) p} E_{2,(2-\alpha) p+1}^{(p+1)}\left(-\lambda_{n} t^{2}\right)\right. \\
& +b \sum_{p=0}^{\infty}(-b)^{p} t^{(2-\alpha)(p+1)} E_{2,(2-\alpha)(p+1)+1}^{(p+1)}\left(-\lambda_{n} t^{2}\right) \\
& \left.+u \sum_{p=0}^{\infty}(-b)^{p} t^{(2-\alpha) p+1} E_{2,(2-\alpha) p+2}^{(p+1)}\left(-\lambda_{n} t^{2}\right)\right\} T_{n}^{(0)}\left(0_{+}\right) \sin \left(\frac{n \pi x}{l}\right), \\
& W_{2}(x, t)=\sum_{n=1}^{\infty} \sum_{p=0}^{\infty}(-b)^{p}\left(\mathcal{E}_{0^{+} ; 2,(2-\alpha) p+2}^{-\lambda_{n} ; p+1,1} \widetilde{g}_{n}\right)(t) \sin \left(\frac{n \pi x}{l}\right), \\
& v(x, t)=h_{1}(t)+\frac{x}{l}\left[h_{2}(t)-h_{1}(t)\right] . \\
& \widetilde{g}_{n}(t)=\frac{2}{l} \int_{0}^{l} \tilde{g}(x, t) \sin \left(\frac{n \pi x}{l}\right) d x, \\
& \widetilde{g}(x, t)=g(x, t)+\frac{\partial^{2} v(x, t)}{\partial x^{2}}-\frac{\partial^{2} v(x, t)}{\partial t^{2}}-b C_{*}^{\alpha} w(x, t),
\end{aligned}
$$

where $\lambda_{n}=\frac{n^{2} \pi^{2}}{l^{2}}$ are eigenvalues of the problem, $u=T_{n}^{(1)}\left(0_{+}\right) / T_{n}^{(0)}\left(0_{+}\right), T_{n}^{(0)}\left(0_{+}\right)=$ $\frac{2}{l} \int_{0}^{l} \widetilde{\Theta}(x) \sin \left(\frac{n \pi x}{l}\right) d x, T_{n}^{(1)}\left(0_{+}\right)=\frac{2}{l} \int_{0}^{l} \widetilde{\Phi}(x) \sin \left(\frac{n \pi x}{l}\right) d x$ are Fourier coefficients, $\widetilde{\Theta}(x)=$ $\Theta(x)-\left.v(x, t)\right|_{t=0_{+}}$, and $\widetilde{\Phi}(x)=\Phi(x)-\left.\frac{\partial v(x, t)}{\partial t}\right|_{t=0_{+}}$.

It is easily observed that for $\gamma \rightarrow 2$, the solution which is given in Theorem 3.3 coincides with (41)-(43).

Acknowledgements

Dedicated to Prof. Dr. Abdullah Altın for his seventieth birthday.

\section{Funding}

No funding.

Availability of data and materials

Data sharing not applicable to this article as no data sets were generated or analysed during the current study.

Competing interests

The authors declare that they have no competing interests.

Authors' contributions

Both authors contributed equally to the paper. All authors read and approved the final manuscript.

\section{Publisher's Note}

Springer Nature remains neutral with regard to jurisdictional claims in published maps and institutional affiliations.

Received: 21 November 2018 Accepted: 21 April 2019 Published online: 22 May 2019 


\section{References}

1. Agarwal, R.P., De Andrade, B., Cuevas, C.: Weighted pseudo-almost periodic solutions of a class of semilinear fractional differential equations. Nonlinear Anal. 11, 3532-3554 (2010)

2. Agarwal, R., Hristova, S., O'Regan, D.: Mittag-Leffler stability for impulsive Caputo fractional differential equations. Differ. Equ. Dyn. Syst. (2017). https://doi.org/10.1007/s12591-017-0384-4

3. Nashine, H.K., Arab, R., Agarwal, R.P., De la Sen, M.: Positive solutions of fractional integral equations by the technique of measure of noncompactness. J. Inequal. Appl. 2017, 225 (2017). https://doi.org/10.1186/s13660-017-1497-6

4. Gorenflo, R., Mainardi, F.: Fractional calculus, integral and differential equations of fractional order. In: Carpinteri, A Mainardi, F. (eds.) Fractals and Fractional Calculus in Continuum Mechanics, pp. 223-276. Springer, Wien (1997)

5. Kilbas, A.A., Srivastava, H.M., Trujillo, J.J.: Theory and Applications of Fractional Differential Equations. North-Holland Mathematics Studies, vol. 204. Elsevier, Amsterdam (2006)

6. Baleanu, D., Agarwal, P., Parmar, R.K., Alqurashi, M.M., Salahshour, S.: Extension of the fractional derivative of the Riemann-Liouville. J. Nonlinear Sci. Appl. 10, 2914-2924 (2017)

7. Kıymaz, I.O., Çetinkaya, A., Agarwal, P.: An extension of Caputo fractional derivative operator and its applications. J. Nonlinear Sci. Appl. 9, 3611-3621 (2016)

8. Agarwal, P., Al-Mdallal, Q., Je Cho, Y., Jain, S.: Fractional differential equations for the generalized Mittag-Leffler function. Adv. Differ. Equ. 2018(1), 58 (2018)

9. Agarwal, P., El-Sayed, A.A.: Non-standard finite difference and Chebyshev collocation methods for solving fractional diffusion equation. Phys. A, Stat. Mech. Appl. 500, 40-49 (2018)

10. Baltaeva, U., Agarwal, P.: Boundary-value problems for the third-order loaded equation with noncharacteristic type-change boundaries. Math. Methods Appl. Sci. 500, 3307-3315 (2018)

11. Agarwal, P., Nieto, J.J., Luo, M.-J.: Extended Riemann-Liouville type fractional derivative operator with applications. Open Math. 15(1), 1667-1681 (2017)

12. Agarwal, P., Jain, S., Mansour, T.: Further extended Caputo fractional derivative operator and its applications. Russ. J. Math. Phys. 24(4), 415-425 (2017)

13. Jain, S., Agarwal, P., Kilicman, A.: Pathway fractional integral operator associated with 3m-parametric Mittag-Leffler functions. Int. J. Appl. Comput. Math. 4(5), 115 (2018)

14. Agarwal, P.: Some inequalities involving Hadamard-type k-fractional integral operators. Math. Methods Appl. Sci. 40(11), 3882-3891 (2017)

15. Ruzhansky, M.V., Je Cho, Y., Agarwal, P., Area, l.: Advances in Real and Complex Analysis with Applications. Springer, Singapore (2017)

16. Mehrez, K., Agarwal, P.: New Hermite-Hadamard type integral inequalities for convex functions and their applications. J. Comput. Appl. Math. 350, 274-285 (2019)

17. Mainardi, F.: Fractional relaxation-oscillation and fractional diffusion-wave phenomena. Chaos Solitons Fractals 7 , $1461-1477$ (1996)

18. Mainardi, F.: The fundamental solutions for the fractional diffusion-wave equation. Appl. Math. Lett. 9, 23-28 (1996)

19. Mainardi, F., Pagnini, G.: The Wright functions as solutions of the time-fractional diffusion equation. Appl. Math. Comput. 141, 51-62 (2003)

20. Mainardi, F., Pagnini, G., Gorenflo, R.: Some aspects fractional diffusion equations of single and distributed order. Appl. Math. Comput. 187, 295-305 (2007)

21. Mainardi, F., Paradisi, P.: Fractional diffusive waves. J. Comput. Acoust. 9, 1417 (2001)

22. Agrawal, O.P.: Solutions for a fractional diffusion-wave equation defined in a bounded domain. Nonlinear Dyn. 29, 145-155 (2002)

23. Camargo, R.F., Capelas de Oliveira, E., Vaz, J. Jr: On anomalous diffusion and the fractional generalized Langevin equation for a harmonic oscillator. J. Math. Phys. 50, Article ID 123518 (2009)

24. Hahn, M., Umarov, S.: Fractional Fokker-Planck-Kolmogorov type equations and their associated stochastic differential equations. Fract. Calc. Appl. Anal. 14, 56-79 (2011)

25. Heinsalu, E., Patriarca, M., Goychuk, I., Schmid, G., Hänggi, P.: Fractional Fokker-Planck dynamics: numerical algorithm and simulations. Phys. Rev. E 73, Article ID 046133 (2006)

26. Kou, S.C., Xie, X.S.: Generalized Langevin equation with fractional Gaussian noise: subdiffusion within a single protein molecule. Phys. Rev. Lett. 93, Article ID 180603 (2046)

27. Lutz, E.: Fractional Langevin equation. Phys. Rev. E 64, Article ID 051106 (2001)

28. Mainardi, F., Pironi, P.: The fractional Langevin equation: Brownian motion revisited. Extr. Math. 10, 140-154 (1996)

29. Metzler, R., Barkai, E., Klafter, J.: Anomalous diffusion and relaxation close to thermal equilibrium: a fractional Fokker-Planck equation approach. Phys. Rev. Lett. 82, Article ID 3563 (1999)

30. Metzler, R., Klafter, J.: The random walk's guide to anomalous diffusion: a fractional dynamics approach. Phys. Rep. $339,1-77(2000)$

31. Sandev, T., Tomovski, Z.: Wave equation for a vibrating string in presence of a fractional friction. In: Proceedings in the Symposium on Fractional Signals and Systems, Lisbon, 4-6 November 2009 (2009)

32. Sandev, T., Tomovski, Z., Dubbeldam, J.L.A.: Generalized Langevin equation with a three parameter Mittag-Leffler noise. Physica A 390, Article ID 36273636 (2011)

33. Sandev, T., Tomovski, Z.: Asymptotic behavior of a harmonic oscillator driven by a generalized Mittag-Leffler noise. Phys. Scr. 82, Article ID 065001 (2010)

34. Diethelm, K., Weibeer, M.: Initial-boundary value problems for time-fractional diffusion-wave equations and their numerical solutions. In: Mehaute, A.L., Machado, J.A., Trigeasson, J.C., Sabatier, J. (eds.) Proceedings of the 1st IFAC Workshop on Fractional Differentiations and Its Applications. ENSEIRB, Bordeux (2004)

35. Luchko, Y.: Maximum principle and its application for the time-fractional diffusion equations. Fract. Calc. Appl. Anal. $14,110-124(2011)$

36. Luchko, Y.: Initial-boundary-value problems for the one-dimensional time-fractional diffusion equation. Fract. Calc. Appl. Anal. 15, 141-160 (2012)

37. Momani, S.: General solutions for the space- and time-fractional diffusion-wave equation. J. Phys. Sci. 10, 30-43 (2006) 
38. Odibat, Z.M., Momani, S.: Approximate solutions for boundary value problems of time-fractional wave equation. Appl. Math. Comput. 181, 767-774 (2006)

39. Odibat, Z.M.: A reliable modification of the rectangular decomposition method. Appl. Math. Comput. 183, 1226-1234 (2006)

40. Sandev, T., Metzler, R., Tomovski, Z:: Fractional diffusion equation with a generalized Riemann-Liouville time fractional derivative. J. Phys. A, Math. Theor. 44, Article ID 255203 (2011)

41. Chen, C.M., Lin, F., Turner, I., Anh, V.: A Fourier method for the fractional diffusion equation describing sub-diffusion. J. Comput. Phys. 227, 886-897 (2007)

42. Sandev, T., Tomovski, Z:: The general time fractional wave equation for a vibrating string. J. Phys. A, Math. Theor. 43, Article ID 055204 (2010)

43. Tomovski, Z., Sandev, T.: Effects of a fractional friction with power-law memory kernel on string vibrations. Comput. Math. Appl. 62, 1554-1561 (2011)

44. Yuste, S.B.: Weighted average finite difference methods for fractional diffusion. J. Comput. Phys. 216, 264-274 (2006)

45. Mittag-Leffler, G.M.: Sur la nouvelle function e(X). C. R. Acad. Sci. Paris 137, 554-558 (1903)

46. Wiman, A.: Über den fundamentalsatz in der theorie der funktionen $e_{\alpha}(x)$. Acta Math. 29, 191-201 (1095)

47. Prabhakar, T.R.: A singular integral equation with a generalized Mittag-Leffler function in the kernel. Yokohama Math. J. 19, 7-15 (1971)

48. Rainville, E.D.: Special Functions. Macmillan Co., New York (1960)

49. Srivastava, H.M., Tomovski, Z:: Fractional calculus with an integral operator containing a generalized Mittag-Leffler function in the kernel. Appl. Math. Comput. 211, 198-210 (2009)

50. Tomovski, Z., Hilfer, R., Srivastava, H.M.: Fractional and operational calculus with generalized fractional derivative operators and Mittag-Leffler type functions. Integral Transforms Spec. Funct. 21, 797-814 (2010)

51. Caputo, M.: Elasticita Dissipacione. Zanichelli, Bologna (1969)

52. Podlubny, I.: Fractional Differential Equations. Academic Press, San Diego (1999)

53. Titchmarsh, E.C.: Introduction to the Theory of Fourier Integrals. Chelsea, New York (1937)

\section{Submit your manuscript to a SpringerOpen ${ }^{\circ}$ journal and benefit from:}

- Convenient online submission

- Rigorous peer review

- Open access: articles freely available online

- High visibility within the field

- Retaining the copyright to your article

Submit your next manuscript at $\gg$ springeropen.com 